\title{
Médiévales
}

Langues, Textes, Histoire

71 | automne 2016

Conflits et concurrence de normes

\section{Le Roman d'Aubéron. Une lecture texte/images}

The Roman d'Aubéron : A Text/Image Reading

Julie Grenon-Morin

\section{(2) OpenEdition}

Journals

Édition électronique

URL : https://journals.openedition.org/medievales/7998

DOI : 10.4000/medievales.7998

ISSN : 1777-5892

Éditeur

Presses universitaires de Vincennes

Édition imprimée

Date de publication : 20 novembre 2016

Pagination : $91-108$

ISBN : 978-2-84292-565-9

ISSN : 0751-2708

Référence électronique

Julie Grenon-Morin, «Le Roman d'Aubéron. Une lecture texte/images », Médiévales [En ligne], 71 |

automne 2016, mis en ligne le 20 septembre 2018, consulté le 22 avril 2022. URL : http://

journals.openedition.org/medievales/7998; DOI : https://doi.org/10.4000/medievales.7998 
Julie Grenon-Morin

\section{Le Roman d'Aubéron Une lecture texte/images}

Le Roman d'Aubéron est conservé dans un manuscrit unique (L. II. 14) de la Biblioteca nazionale universitaria de Turin (BNU), imposant recueil de 586 folios où ce roman se trouve entre les folios $283 \mathrm{r}$ et $296 \mathrm{v}$, et où il est suivi par l'ensemble du cycle de Huon de Bordeaux (folios 283r-460v). Composé vraisemblablement entre 1260 et 1311 (date de la rédaction de ce manuscrit), par un auteur anonyme écrivant en dialecte picard, ce récit de 2468 vers est copié sur deux colonnes et est accompagné de trois miniatures (folios 283, 289 et 291). Celles-ci n'ayant jamais été reproduites jusqu'à aujourd'hui, nous entendons ajouter à la belle édition critique publiée en 1973 par Jean Subrenat une étude consacrée aux rapports entre le texte et l'image ${ }^{1}$. Les clichés que nous présentons de ces images rendent compte de l'état du manuscrit où elles se trouvent : ce recueil a en effet été dégradé par le feu lors de l'incendie de la BNU en janvier 1904. Avant cet incendie, ce roman avait été édité en 1878 par Arturo Graf ${ }^{2}$. Fondée sur un codex qui était encore intact, cette publication est indispensable pour toute étude consacrée à ce texte.

Comme le notent Sandrine Hériché-Pradeau et Maud Pérez-Simon, « regarder l'image peut inciter le lecteur médiéval ainsi que le lecteur moderne à reconsidérer le texte et à en réévaluer la porté $\mathrm{e}^{3} »$. Créations d'un miniaturiste anonyme, les illustrations du Roman d'Aubéron ont une fonction à la fois décorative et pédagogique. Elles servent notamment à

1. Le Roman d'Aubéron, éd. J. Subrenat, Paris/Genève, 1973. Toutes les citations sont tirées de cette édition.

2. A. GRAF, I complementi della chanson d'Huon de Bordeaux. Testi francesi tratti da un codice della Biblioteca di Torino..., t. I, Auberon, Halle, 1878. Cette édition a fait l'objet de deux importants comptes rendus parus la même année, l'un par G. PARIS, Romania, 2 (1878), p. 332-339, l'autre par A. STIMming, Zeitschrift für romanische Philologie, 2 (1878), p. 609-616.

3. S. Hériché-Pradeau et M. Pérez-Simon éd., Quand l'image relit le texte. Regards croisés sur les manuscrits médiévaux, Paris, 2013, p. 11. 
découper un texte au "schéma compliqué ${ }^{4}$ », qui enchaîne des " rencontres insolites » et des "péripéties dramatiques, souvent teintées d'une sorte de boursoufflure à tonalité burlesque $»^{5}$. Les images des folios 283 et 291 paraissent les plus complexes, tandis que la force esthétique de celle du folio 289 s'avère frappante. Nous verrons que ces trois miniatures «accompagne[nt] et nourri[ssent] le texte ${ }^{6} »$.

Incrustées de feuilles d'or, ces miniatures sont rehaussées d'images en marge représentant des végétaux, des personnages et des animaux. « Véritable espace de liberté, laissé ouvert à l'imagination des enlumineurs, mais non dénué de sens ${ }^{7}$ », l'image marginale «trahit tout à la fois une angoisse de la classification et un souci de ne rien signifier afin que le centre puisse être le lieu du sens ${ }^{8} »$. Nous interrogerons également les liens que ces marginalia peuvent entretenir avec le texte qu'elles côtoient.

Ces trois miniatures ont souffert du feu qui a ravagé le manuscrit. Les pages sont déchirées à l'endroit des reliures. Les bords noircis des folios expliquent pourquoi la première miniature (folio 283) est déformée (fig. 1). C'est la moins bien conservée des trois, avec ses feuilles d'or et ses couleurs endommagées. Il en est de même pour les marges de ce feuillet où l'on distingue deux personnages à cheval et des fleurs (marge inférieure), une branche avec un oiseau (marges droite et gauche). Au bas de la page, on voit enfin deux centaures (fig. 2). Sur le côté a été peint un oiseau sur le haut d'une branche. Un texte en rouge (comme pour les deux autres miniatures) se trouve au-dessus de cette dernière, en lettres rubriquées. Le côté gauche de la miniature a subi plus fortement que le droit les dommages de l'incendie et la feuille d'or y est plus effritée également.

La miniature du folio 289 est la mieux conservée (fig. 3). Les couleurs et l'or sont parfois abîmés, notamment le cadre. L'image est en effet entourée d'un cadre rouge et bleu, avec des carrés dorés aux quatre coins. Ceci n'est pas surprenant puisqu'à l'époque une image est "généralement enfermée dans un cadre ${ }^{9} »$. De fins motifs blancs ont aussi été rajoutés aux lignes du cadre (c'est également le cas pour la première miniature).

Le folio 291 a subi les mêmes dégradations que le folio 283 pour ce qui concerne les feuilles d'or et les couleurs (fig. 6). Les illustrations de la marge inférieure sont partiellement effacées. La cape bleue d'un des deux personnages à cheval a presque entièrement disparu (fig. 5). On remarque

4. C. Roussel, «Judas Maccabée, Jules César,Arthur, saint Georges et les moustaches de saint Joseph... De quelques rencontres improbables dans le Roman d'Aubéron », dans J.-P. Dubost éd., Topographie de la rencontre dans le roman européen, Clermont-Ferrand, 2008, p. 55.

5. Ibid., p. 57.

6. S. Cassagnes-Brouluet, La Passion du livre au Moyen Âge, Rennes, 2010, p. 7.

7. Ibid., p. 94.

8. M. Camille, Images dans les marges. Aux limites de l'art médiéval, Paris, 1997, p. 69.

9. F. BARBIER, Histoire du livre, Paris, 2006, p. 66. 
aussi, d'après la reproduction, que le bleu des ornementations est généralement plus pâle que celui de la miniature, ce qui laisse croire que cette couleur s'est estompée avec le temps; c'est aussi le cas du personnage en bas à droite en tenue mauve (probablement plus pâle qu'à l'origine) et dont le visage est en partie effacé.

Malgré l'état dans lequel se trouvent ces trois miniatures, elles comprennent des indices iconographiques offrant des pistes de lecture intéressantes que le texte seul du Roman d'Auberon ne permet pas de déceler. Avant de les analyser, cependant, il nous faut encore résumer brièvement le Roman d'Aubéron ${ }^{10}$. Rappelons tout d'abord qu'il s'agit d'une sorte de récit généalogique servant de «prequel » à la chanson de gestes consacrée à Huon de Bordeaux - comme en témoigne le manuscrit où il se trouve contenu ${ }^{11}$. Plusieurs actions et temporalités en apparence disparates composent ce récit tiré en partie de l'ouvrage qu'il entend introduire : « tantôt il élague et en retient ce qu'exploitera l'intrigue à venir, tantôt au contraire il amplifie et multiplie les détails ${ }^{12} »$. La composition fantaisiste, qui mêle tradition hagiographique «folklorisée ${ }^{13}$ » et héritage épique, relève de plus d'une « aptitude boulimique à intégrer les matériaux narratifs les plus hétéroclites ${ }^{14} »$.

Le personnage initial se nomme Judas Machabée, un homme pourvu de «nombreuses qualités ». Le roi Brandifort, jaloux de lui, décide de l'attaquer et fait le siège de ses terres. Guidé par un songe, Judas provoque le roi et le tue, comme l'illustre la première miniature du folio 283. Il s'empare alors de ses biens et épouse sa fille. Le couple donne naissance à une fille, Brunehaut, bénie par quatre fées. L'une d'elles émet un vœu, à savoir que la future jeune fille quitte un jour sa famille pour rejoindre le royaume de Féérie. Sept ans plus tard, Brunehaut s'y rend et obtient des pouvoirs magiques. Elle épouse Césaire et ils ont ensemble un fils : Jules César. Césaire se rend ensuite en Hongrie, muni d'un haubert magique, pour terrasser le géant Brulant, scène illustrée dans la deuxième miniature (folio 289). Césaire veut voir ce dernier épouser la fée Morgain, sœur du roi Arthur. De cette union naissent des jumeaux, Aubéron et Georges, et trois fées formulent à nouveau à leur naissance des vœux, six en tout (ce qu'illustre la troisième miniature : folio 291). Selon le souhait de l'une d'elles, Aubéron cessera de grandir quand il atteindra la hauteur de trois

10. Ce Roman est divisé en cinquante-et-un passages, chacun annoncé par des chiffres romains, que J. Subrenat a regroupés en sept parties : «Judas », « Enfances Brunehaut », «Le Cerf », « Enfances Jules César », « "Enfances” des Jumeaux », « Georges » et « Auberon ».

11. Huon de Bordeaux. Chanson de geste du XIII siècle, publiée d'après le manuscrit de Paris BnF fr. 22555, éd. W. W. KLiber et F. Suard, Paris, 2003.

12. C. Roussel, « Judas Maccabée, Jules César, Arthur... », p. 55.

13. Ibid., p. 60.

14. Ibid., p. 62. 
pieds. Morgain lui fait présent d'un cor magique. Aubéron reçoit également un épervier et un haubert merveilleux. Quant à Georges, après plusieurs péripéties, il succède à Jules César. Aubéron vit plus de cent ans dans la ville de Montmur. Après qu'on lui a dérobé le haubert magique, il est chassé de son trône par le géant L'Orgueilleux. Brunehaut prédit alors la venue de Huon de Bordeaux qui reprendra le précieux objet.

\section{Ch'est li commencemens dou roumant d'Auberon...}

La rubrique ${ }^{15}$ en rouge de la première miniature (fig. 1), la plus complexe des trois, marque le tout début du roman (f. 283r) ${ }^{16}$. Comme c'est souvent le cas, elle fait « office de frontispice ${ }^{17} »:$ : Ch'est li commencemens dou roumant d'Auberon que ses aves Judas Machabeus fu assis a Macabé dou roy Bandifort. » La miniature forme une sorte de triptyque qui trace une chronologie unissant le passé, le présent et le futur. L'image est divisée en deux par le château qui s'élève sur toute la hauteur du centre de l'image, semblable à une tour : d'une part une étendue d'eau, d'autre part une scène de bataille. Judas, couronné avant sa victoire, est figuré par deux fois dans la tour (en rouge avec un collet bleu). À gauche, depuis un rempart, il observe la nature et se laisse guider par les signes de son rêve. À droite, il apparaît à travers l'une des fenêtres de la demeure fortifiée. Le château est exagérément haut et on peut apercevoir deux entrées entièrement noires avec des herses levées. L'une de ces entrées est entourée de tourelles au pignon bleu. Les trois temps sont mis en évidence par le cadre passant du bleu au rouge puis à nouveau au bleu. Tout le ciel est recouvert d'or.

Dans le premier tiers de l'image, nous apercevons un arbre ainsi qu'un lac avec des oiseaux. Ceux-ci revêtent une signification symbolique particulière : « les oiseaux sont bien représentés dans le matériel symbolique, peut-être aussi parce qu'entrant dans des pratiques divinatoires, ils ont une place quasi naturelle dans des songes à valeur oraculaire ${ }^{18} »$. On perçoit un rapace (un autour) et un canard, illustrant le rêve de Judas qui le convainc de livrer bataille.

«On sait que les songes des personnages épiques sont souvent peuplés d'animaux ${ }^{19}$. »C'est le cas du Roman d'Aubéron. La symbolique des oiseaux n'a guère besoin d'être décodée, puisque l'histoire fait elle-

15. Jean Subrenat utilise le terme de « légende » (p. LXXV).

16. Les trois rubriques proviennent de l'édition de J. Subrenat (p. LLXV), qui tient ces informations de l'édition d'A. Graf puisque le feu a rendu la lecture entre-temps impossible.

17. F. BARBIER, Histoire du livre, p. 67.

18. M. Demaule, La Corne et l'ivoire : étude sur le récit de rêve dans la littérature romanesque des XII et XIII siècles, Paris, 2010, p. 415.

19. C. Heck et R. Cordonnier, Le Bestiaire médiéval : l'animal dans les manuscrits enluminés, Paris, 2011, p. 116. 


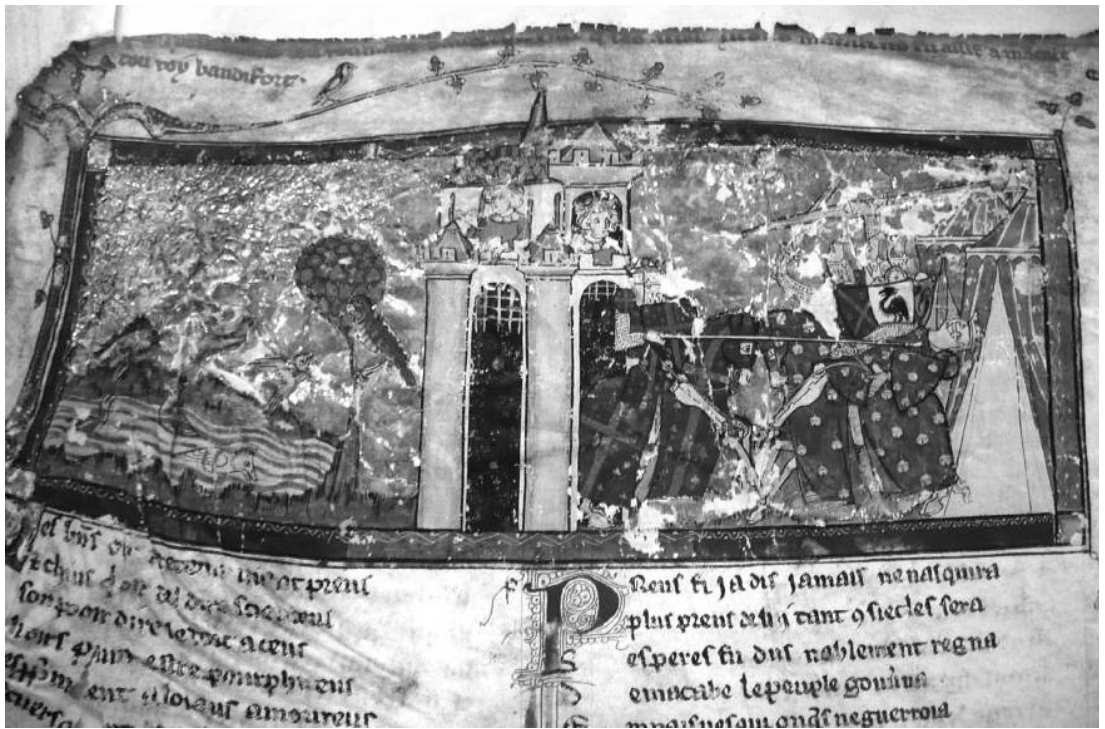

Fig. 1. Frontispice : Judas (Torino, BNU, ms. L. II. 14, f. 283r).

même mention de Judas et de l'interprétation de son rêve qui le mène sur le champ de bataille. De manière attendue, le songe de Judas nous informe sur la suite de l'histoire ${ }^{20}$, tout en comprenant des images énigmatiques ${ }^{21}$. Le rêve a en effet une fonction d'incubateur préalable au récit, comme l'affirme Fabienne Pomel, puisqu'il fait « obtenir de la divinité une information ${ }^{22}$ » qui sera développée par la suite. L'épisode onirique du personnage s'inscrit dans une symbolique qui se fonde non seulement sur les choses de la Nature, mais aussi dans une tradition spirituelle marquée par des courants païens, profanes, gnostiques ou judéo-chrétiens ${ }^{23}$.

L'arbre sert de perchoir à l'oiseau de proie, le choix du rapace et du canard comme volatiles correspond à la vision de Judas : « Sur son vivier un grant arbre esgarda. / Vit un ostoir fourmé qui manoit la ; / Vit qu'il miautit, vers l'iaue s'envolla. / I. grant malart saisi et empieta / A la terre l'ocist et devora » (v. 111-115). Impétueux, rapide et précis, le rapace est « un symbole de domination et de puissance ${ }^{24}$ » et manifeste habituellement « une présence hostile $^{25} »$. L'histoire raconte qu'un oiseau de proie tue une bête inoffensive.

20. H. Braet, Le Songe dans la chanson de geste au XII siècle, Gand, 1975, p. 111.

21. Ibid., p. 113.

22. F. Pomel, «Songes d'incubation et incubations de l'œuvre », dans C. FerLampinAcher, E. Gaucher et D. Hüe éd., Perspectives médiévales. Actes du colloque Sommeil, songes et insomnies, Paris, 2008, p. 109.

23. H. BRAEt, Le Songe dans la chanson de geste..., p. 114.

24. M. Demaule, La Corne et l'ivoire..., p. 416.

25. H. Braet, Le Songe dans la chanson de geste..., p. 132. 
Le rapace, de couleur noire, est plus gros que ses congénères et observe les plus petits, tandis que d'autres bêtes à plumes dans les tons de blanc et de gris volent vers lui. Si les caractéristiques des oiseaux relèvent généralement de la tradition des bestiaires, leur couleur peut aussi contribuer à en souligner la valeur symbolique. Mais, alors que le noir est traditionnellement considéré comme une couleur « diabolique »par opposition au blanc qui est une « couleur divine $»^{26}$, cette symbolique est ici inversée : le noir est devenu une « couleur honnête ${ }^{27}$ », tandis que le blanc « devient un faux-semblant du noir, une couleur non plus divine mais trompeuse ${ }^{28} »$. L'agression du rapace a en effet un sens de régénération : Judas s'attaque à son ennemi et décuple son pouvoir par sa victoire. Malgré ses traits habituellement négatifs, le rapace possède donc ici une valeur positive. Pouvant également symboliser l'ascension vers le ciel $^{29}$, il annonce notamment le prochain succès de Judas.

L'oiseau à l'horizontale dans l'eau, le bec ouvert et mort, quoique aucun sang ou blessure n'est visible, est un canard. Autour de lui, l'eau est représentée par des stries bleues en mouvement ondulé et est entourée de pâturage. Décrit comme grand, le canard est la proie de l'autour qui le tue et le dévore. Sa symbolique est moins bien étudiée que celle des rapaces. Quoiqu'ils fassent partie du quotidien des gens du Moyen Âge, du fait qu'il s'agit d'un animal de basse-cour, les canards sont peu représentés dans les miniatures et, lorsqu'ils le sont, c'est généralement dans les ornementations. La Bible n'en fait pas mention non plus et c'est ce qui pourrait expliquer le désintérêt des exégètes à son endroit ${ }^{30}$. Dans ce manuscrit, le canard est peu réaliste et cette incongruité laisse à penser que les artistes éprouvaient de la difficulté à reproduire ce type animalier, peut-être par manque d'habitude.

La triple présence de Judas Macchabée dans la miniature souligne l'importance de ce personnage. Il apparaît à droite de l'image, habillé en bleu $^{31}$ et victorieux de Brandifort, ce qui fait de lui un personnage actif. La couleur bleue connote des personnages « courageux, loyaux, fidèles ${ }^{32}$ ». Le mouvement de la lance effectué par Judas est clair : il est vainqueur de son ennemi et est en train de le terrasser. Le premier et le troisième tiers sont donc en opposition : beaucoup de vivacité à gauche, contrairement à

26. M. Demaule, La Corne et l'ivoire..., p. 418.

27. M. Pastroureau, d'après M. Demaule, La Corne et l'ivoire ..., p. 419.

28. M. Demaule, La Corne et l'ivoire..., p. 418.

29. J. Dalarun, Le Moyen Âge en lumière : manuscrits enluminés des bibliothèques de France, Paris, 2002, p. 88.

30. C. Heck et R. Cordonnier, Le Bestiaire médiéval..., p. 117.

31. À cette époque, "la civilisation occidentale est devenue une civilisation du bleu », selon M. PAStOUREAU, « Une histoire des couleurs est-elle possible ? », Ethnologie française, 20/4 (1990), Paradoxes de la couleur, p. 369.

32. M. Pastoureau. Le Bleu : histoire d'une couleur, Paris, 2000, p. 60. 
la partie de droite, plus calme et dénuée d'éléments. À l'extrême droite de l'image sont figurées des tentes rouges et vertes.

Par sa position élevée, Judas est présenté comme un homme de pouvoir : un pouvoir exercé sur la Nature et sur l'issue du combat. Judas observe des éléments propres au calme (oiseaux, lac, prairies), alors que le second contemple l'action d'une bataille. Celle-ci met l'accent sur Brandifort transpercé par une lance : «D'une lance le va grant cop paier ; / Le fort escu li fait ronpre et percier ; / Hauberc ot bon, ne pot maille empirier, / Ançois convint la lance perçoiier »(v. 161-164). Sur l'illustration, la lance fait basculer l'ennemi, mais on ne voit pas de sang. De plus, le regard de Judas délimite deux temps très opposés, des moments-clés dont il est l'instigateur : après tout, il est à l'origine de la lignée qui conduira à Aubéron. Judas, dans sa tour et sur le champ de bataille, est donc représenté dans deux états, montrant ainsi plusieurs moments du personnage.

La position centrale du château exprime sa valeur et sa puissance : « [1] es imagiers utilisent volontiers la fortification comme symbole de la solidité et des réalités spirituelles ${ }^{33}$ ». Sous la herse levée, le cheval de Judas n'est pas tout à fait sorti du château, ce qui laisse peut-être penser à un combat bref et facile : "Par une porte, au lés, vers le vivier, / S'en ist Judas armés sur son destrier » (v. 134-135). L'architecture improbable du bâtiment s'explique par une difficulté à prendre en compte l'échelle. Bien qu'il faille accorder une part non négligeable à la symbolique, cette difficulté est récurrente dans les images médiévales et s'explique par une méconnaissance des lois de la perspective qui rend erronées les représentations des distances et des angles visuels ${ }^{34}$. Les distances ne sont pas réalistes non plus, notamment la proximité des tentes de l'ennemi.

Il faut également noter les armoiries et les couleurs des deux camps, soit le rouge et le bleu. Judas arbore la croix chrétienne et la fleur de lys dorée sur fond bleu azur, couleur valorisée en héraldique. Ces symboles attestent ses croyances religieuses. Selon Michel Pastoureau, le bleu a été promu dès le XII ${ }^{\mathrm{e}}$ siècle : « il devient rapidement une couleur à la mode, une couleur aristocratique, et même déjà la plus belle couleur selon certains auteurs ${ }^{35}$ ». Cette valorisation « touche tous les domaines de la vie sociale, agit profondément sur les modes de sensibilité et a des conséquences économiques importantes ${ }^{36} »$. Toujours selon Michel Pastoureau, le bleu et le rouge sont d'ailleurs des couleurs en constante opposition depuis la montée en puissance du bleu, provoquée par un avancement dans les

33. Ibid.,p. 195.

34. J. Wirth, L'Image à la fin du Moyen Âge, Paris, 2011, p. 360.

35. M. Pastoureau, Le Bleu..., p. 49.

36. ID., Jésus chez le teinturier. Couleurs et teintures dans l'Occident médiéval, Paris, 1997, p. 104. 
techniques de teinture ${ }^{37}$. Elles répondent ici à une héraldique imaginaire. Le rouge, qui était à une certaine époque la couleur la plus prestigieuse, « celle autour de laquelle s'articulaient tous les systèmes symboliques et tous les codes sociaux construits sur l'univers des couleurs ${ }^{38} »$, perd du terrain. En effet, à l'époque de la création du manuscrit, le rouge est employé le plus souvent pour prêter de mauvaises intentions à un personnage ${ }^{39}$.

Brandifort et son cheval arborent une couleur rouge sang avec des motifs qui semblent être des noisettes de couleur or. Un dragon, relevant d'une héraldique imaginaire, est aussi visible sur un des écus de la mêlée. Le chevalier qui se sert de l'écu semble diriger son épée vers Judas ; nous pouvons donc penser qu'il appartient au parti de l'ennemi. Il faut aussi noter que la peinture dorée remplace partout le bleu du ciel.

De façon générale, cette première image en trois volets qui ouvre le Roman d'Aubéron est fidèle au récit. L'action de Judas est parfaitement illustrée : «D'ont point vers lui, n'ot soing de manecier. / D'une lance le va grant cop percier » (v. 159-160). Le miniaturiste s'est efforcé de demeurer très proche de l'histoire, sans se livrer à une interprétation personnelle, puisque de nombreux éléments sont représentés : les plus évidents, comme les allusions au rêve, mais aussi certains plus anodins comme la porte. Évidemment, le miniaturiste a dû hiérarchiser les évènements et n'en conserver que quelques-uns ; sont évacués par exemple l'écu percé de l'ennemi ou celui-ci décédé.

Dans les marges inférieures (fig. 2), deux centaures illustrent le talent de Judas sur une monture ainsi que le ton merveilleux du texte dans son ensemble. Au-dessus de la miniature a été peint un oiseau perché sur une branche, un rappel de la miniature ou une simple décoration à vocation esthétique. Les centaures apparaissent comme une « déformation volontaire du texte ${ }^{40} »$, soit comme sa réappropriation et sa transformation par les miniaturistes. Ils viennent corroborer l'aura magique du Roman, bien que celui-ci n'en fait guère mention dans ses vers. Il s'agit en quelque sorte d'une relecture invitant le lecteur à saisir le caractère surnaturel de l'œuvre. La mise en marge signifie aussi qu'il n'est pas absolument prépondérant dans l'histoire et que le religieux et le réalisme sont d'une importance supérieure. En ce qui concerne les oiseaux, ils semblent offrir les éléments d'un savoir rudimentaire sur le monde ${ }^{41}$. Leur présence ponctuelle aurait donc une valeur didactique et esthétique.

37. Ibid., p. 108-112.

38. M. Pastoureau, « Une histoire des couleurs est-elle possible ?»..., p. 369.

39. ID., Le Bleu..., p. 59.

40. M. CAMiLle, Images dans les marges..., p. 58.

41. M. Carruthers, The Book of Memory : a Study of Memory in Medieval Culture, Cambridge, 1990, cité d'après J. WIRTH, Les Marges à drôleries des manuscrits gothiques (1250-1350), Genève, 2008, p. 144. 


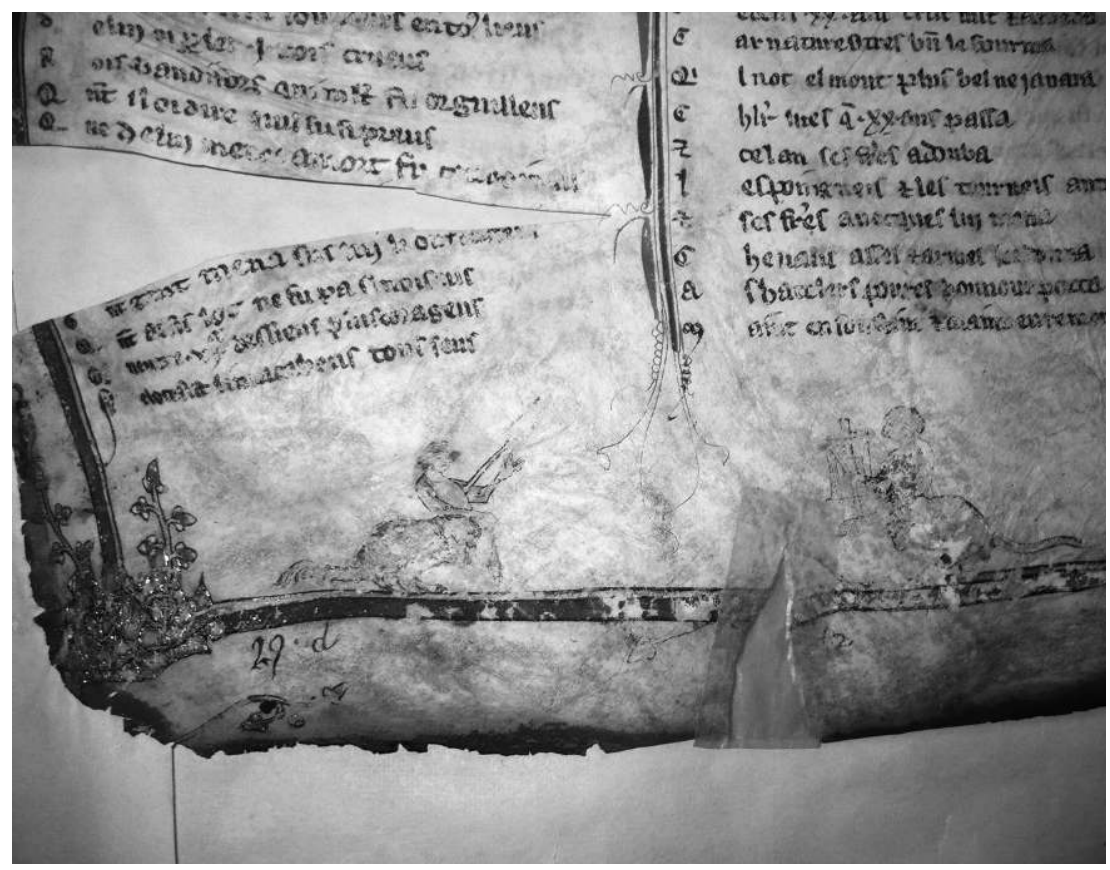

Fig. 2. Image marginale : deux centaures (Torino, BNU, ms. L. II. 14, f. 283r).

\section{Ensi que Jules Chezaires se conbati...}

La deuxième miniature (fig. 3) est accompagnée d'une rubrique, située au haut du feuillet (folio 289) : «Ensi que Jules Chezaires se conbati au grant gaiiant qui fu taiions a l'Orgueliex qui puis tauli Auberon sen fill le hauberc et le tour de Dunostre. » Elle représente une autre scène de guerre, où les belligérants sont plus nombreux. Le mouvement particulier de la figuration est créé par les multiples épées dans les airs qui s'abattent sur le géant Brulant. Les lances donnent un effet de dynamisme supplémentaire à cette image. On compte cinq chevaux ainsi que des chevaliers au nombre de douze, en dénombrant celui étendu en premier plan.

Les chevaliers sont tour à tour drapés de bleu, de rouge ou de jaune orange. Certains ont des heaumes verts. Le jaune devient une couleur de plus en plus étrange entre le haut et le bas Moyen $\hat{A} g e^{42}$. La juxtaposition du vert et du jaune exprime la violence, en raison d'un ordre des couleurs; au Moyen Âge, le contraste entre ces deux teintes est le plus grand qui puisse exister : côte à côte, elles sont toujours signe « de désordre, d'agressivité, de

42. M. Pastoureau, Jésus chez le teinturier..., p. 152. 


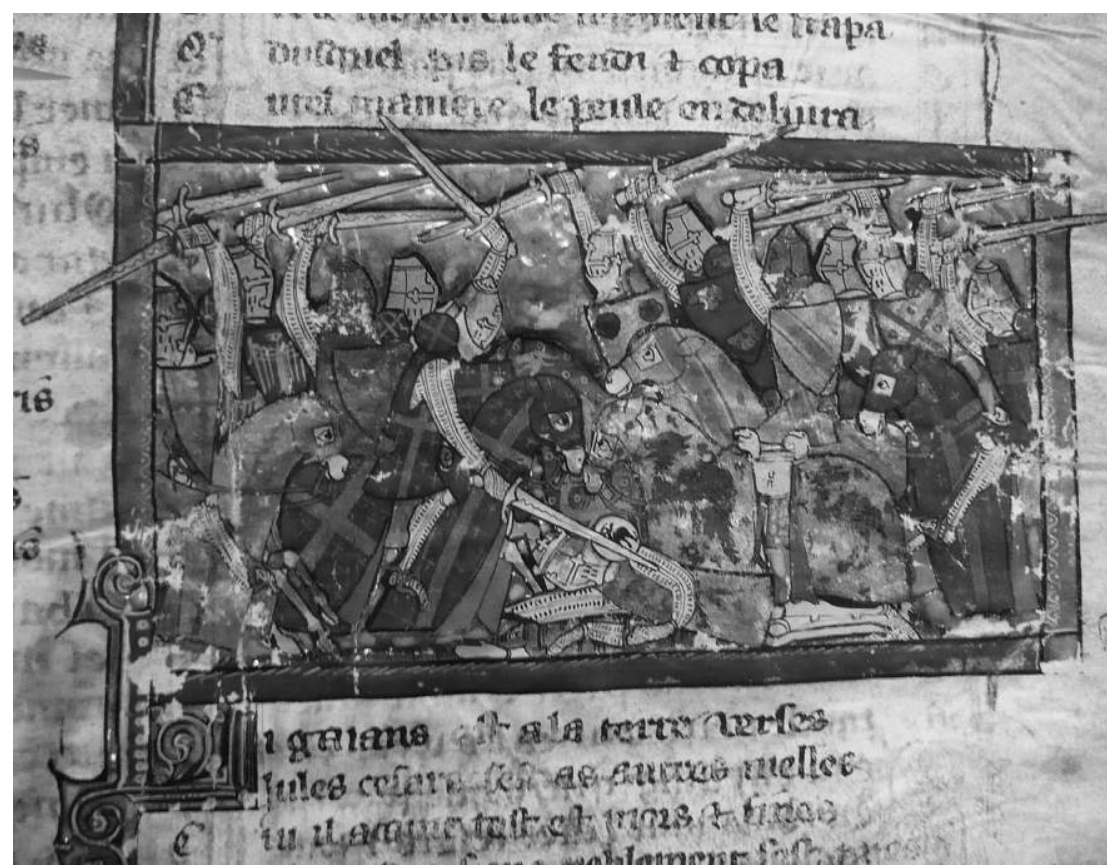

Fig. 3. Deuxième miniature : scène de combats avec, à terre, le géant Brulant (Torino, BNU, ms. L. II. 14, f. 289r).

déraison ${ }^{43} »$. Les destriers en bleu arborent encore la croix chrétienne et la fleur de lys. Celui de gauche, en bleu, tout comme la housse de son cheval, est forcément Jules César puisqu'il frappe de son épée le géant.

On distingue six têtes de chevaux, mais on en devine d'autres dissimulées. En tant que montures de guerre, les animaux de cette image font partie des représentations les plus fréquentes dans les enluminures ${ }^{44}$. Ils connotent la puissance et la richesse, cette dernière étant la plus grande des vanités ${ }^{45}$. Les chevaux de guerre sont souvent indissociables des récits épiques, ce que relate Marina Viallon dans un article récent. L'auteure y évoque la riche symbolique qui entoure cet animal de grande valeur : en effet, un « cheval est un animal cher à l'achat et à l'entretien, d'autant plus, que contrairement aux autres gros animaux domestiques, il ne produit ni lait ni laine et la consommation de sa viande est officiellement proscrite par l'Église ${ }^{46} »$.

43. Ibid., p. 162.

44. C. Heck et R. Cordonnier, Le Bestiaire médiéval..., p. 311.

45. Ibid., p. 308.

46. M. Viallon, «Fiers destriers : images du cheval de guerre au Moyen Âge », In Situ, 27 (2015), mis en ligne le 2 novembre 2015 : http://insitu.revues.org/12066 ; DOI 10.4000/ insitu.12066 [consulté le 5 avril 2016]. 
Les robes des chevaux, pas très visibles, sont toutes ici de couleur pâle : les deux montures les plus à gauche et celle de Brulant sont de couleur châtain, alors que les deux autres, vers la droite, sont blanches. Aucune des bêtes n'est de couleur cendrée - la moins appréciée pour un cheval - ou de couleur baie, qui évoque souvent la puissance. Le regard des chevaux a quelque chose d'humain, de doux et de sensible, ce qui rappelle l'attachement et l'importance du cheval, animal aristocratique par excellence.

Contrairement à celles des autres chevaliers, la housse du cheval de Brulant est grise. Elle est ornée avec ce qui semble être un dragon. Le géant arbore cette même teinte de gris, mais le motif brun de sa cuirasse est effacé et n'a laissé que quelques traces impossibles à définir. Sur l'épaule droite de son armure, on aperçoit le même motif animalier de loup. Cet animal incarne «toutes les craintes humaines vis-à-vis du monde animal ${ }^{47}$ » et la véritable nature du personnage, considéré comme un loup. Le cheval du géant a la tête légèrement plus basse que celui de Jules César, comme un signe de soumission au vainqueur. Ces deux montures échangent un regard d'où se dégage une certaine émotion, presque de la pitié pour leur situation respective de serviteur.

Abattu au premier plan, le géant Brulant est, malgré cette caractéristique, à peu près de la même grandeur que les chevaliers qui sont debout, et cela dans le but de gratifier le vainqueur. Aussi, est-il le seul dont l'arme porte un coup : «Jules Cesars de son branc l'assena, / Droit sus son elme telement le frapa / Que dusqu'el pis le fendi et copa » (v. 11101112) ; toutes les autres armes sont en mouvement dans les airs. C'est à ce personnage anachronique que revient tout l'honneur de la victoire en Hongrie. L'image ne laisse cependant pas voir que le heaume de l'ennemi est fendu comme l'indiquent les vers. Le texte mentionne que « tous li cans en est ensanglentés » (v. 1119), mais il n'y a pas de sang dans l'image. Alors que le texte souligne la violence, les miniatures mettent davantage l'accent sur les faits d'armes et le reste de l'histoire.

On ne trouve pas ici d'oiseau, mais seulement des branchages et des feuillages, ce qui renforce ici l'idée selon laquelle les oiseaux du folio précédent se voulaient un rappel de la miniature. Deux figures terminent chacune des deux colonnes du texte : il s'agit de deux singes en pleine action (fig. 6). Celui de gauche manie un tissu rouge dans les airs; celui de droite tire à l'arbalète.

Les singes sont de loin les animaux les plus représentés dans les marges des manuscrits. Ils permettent de présenter « une imitation difforme de l'être humain qui symbolise parfaitement la perte de la ressemblance

47. C. Heck et R. Cordonnier, Le Bestiaire médiéval..., p. 387. 


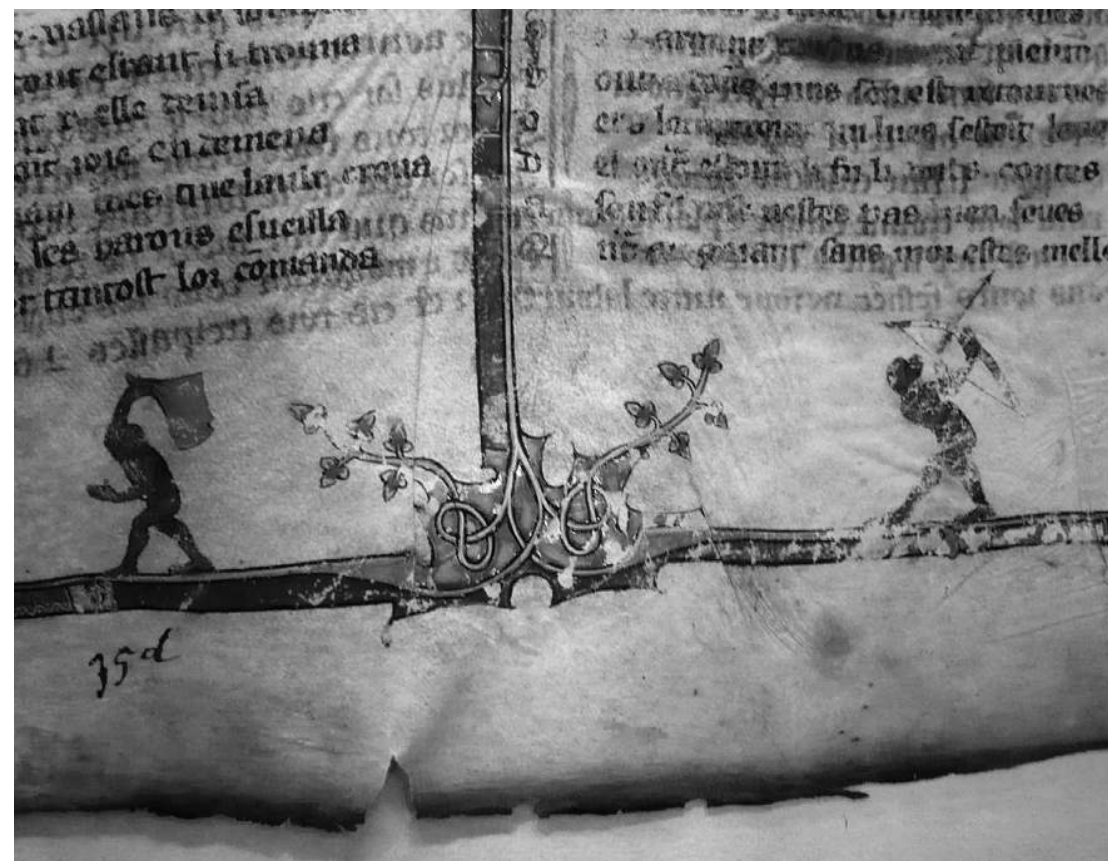

Fig. 6. Image marginale : deux singes (Torino, BNU, ms. L. II. 14, f. 289r).

divine, due à la chute de nos premiers parents ${ }^{48} »$. Figure parodique, « le singe représente donc les instincts de l'homme que ce dernier doit dompter pour retrouver la noblesse de sa nature ${ }^{49}$ ». Deux hypothèses peuvent être avancées pour expliquer la présence de ces deux singes, toutes deux légitimes. D'une part, ils se moquent de la bataille, fait d'armes sanglant qui s'éloigne de l'idéal de paix chrétien. D'autre part, ils ne se rient que de l'ennemi, c'est-à-dire le géant Brulant. Même si la deuxième hypothèse semble la plus probable, il paraît impossible de trancher. Les singes se substituent aux combattants et s'accaparent leurs faits et gestes. Ils ont comme fonction prédominante de commenter l'image principale, comme c'est généralement le cas par ailleurs, afin « donner une image complète du monde, faite de lumières et d'ombres ${ }^{50} \gg$. Un lecteur attentif serait donc à même de comprendre que les guerres ne sont pas tout à fait morales ou bien que Brulant est un être facile à combattre, maladroit, ridicule.

48. J. Wirth, Les Marges à drôleries..., p. 313. Voir aussi M. CAmille, Images dans les marges..., p. 21.

49. C. Heck et R. Cordonnier, Le Bestiaire médiéval..., p. 538.

50. J. WiRTH, Les Marges à drôleries..., p. 362. 


\section{Einsi que Morgue le fee...}

La troisième miniature (fig. 4) est expliquée par la didascalie suivante, située au haut du feuillet (f. 291) : «Einsi que Morgue le fee s'agut a Monmur de S. Jorge et d'Auberon le faé et ensi que les fees leur donnerent les dons au naistre. » Contrairement aux deux autres miniatures, le cadre constitue un rempart à quatre tourelles bleues, encore un symbole de puissance, qui souligne l'importance de la scène. Cette image illustre, sur la gauche, la naissance du roi Aubéron et, sur la droite, les trois fées lui prodiguant ses dons merveilleux. Le couvre-lit bleu, parsemé d'étoiles dorées, qui orne le lit de l'accouchée occupe presque les deux tiers de l'image ; cette couleur montre l'importance hiérarchique du personnage. Une draperie verte (déteinte) surplombe le lit et deux chandeliers reposent sur une étagère située au-dessus des six personnages adultes à droite.

Dans des draps immaculés, la mère est habillée tout de blanc, comme l'est d'ailleurs la peau de son visage et de ses mains (il s'agit probablement du même pigment utilisé par le peintre) : ce type de teint constitue habituellement un signe de beauté. Une reine ne saurait être laide, surtout dans un récit où l'on veut encenser une dynastie. Assise sur son lit, elle retient George, l'aîné, par le bras, alors que l'une des fées tire le bébé vers elle. Il ne peut pas y avoir de méprise sur l'identité des jumeaux : «Le permier né l'une d'eles pris a, / Et le mainsné la seconde combra ; / D'iax aiisier cascune se pena : / Moult volentiers la tierce lor aida » (v. 13611364). De plus, George est plus gros que son frère. L'aîné et futur empereur est nu et, comme s'il ne voulait pas quitter sa mère, il a la tête tournée vers elle et la regarde.

Trois figures féminines entourent Aubéron emmailloté dans une couverture blanche et verte; celle qui se trouve le plus à droite le tient dans les bras. On pourrait penser que nous avons là les trois fées occupées à discuter des dons dont elles pourvoiront les enfants, l'une d'elles se trouvant dédoublée. Il pourrait s'agir de n'importe quelle personne de la suite, étant donné la teinte de son vêtement qui semble indiquer son appartenance au lignage noble. Ces quatre femmes ne doivent donc pas être confondues : les trois fées ont la même coiffure et le même type de robe, alors que le voile de l'autre femme crée un contraste. Le tableau suivant permet de résumer ce que dit le texte au sujet des vœux formulés par les trois fées :

\begin{tabular}{|c|c|c|}
\hline & Georges (l'aîné) & Aubéron (le cadet) \\
\hline Fée 1 (lilas, tient Georges) & sera empereur & mesurera 3 pieds \\
\hline Fée 2 (rouge, tient Aubéron) & épousera une noble dame & couronne de Monmur \\
\hline Fée 3 (rose, aide Fée 2) & sera sanctifié au Paradis & vivra 400 ans, beauté \\
\hline
\end{tabular}




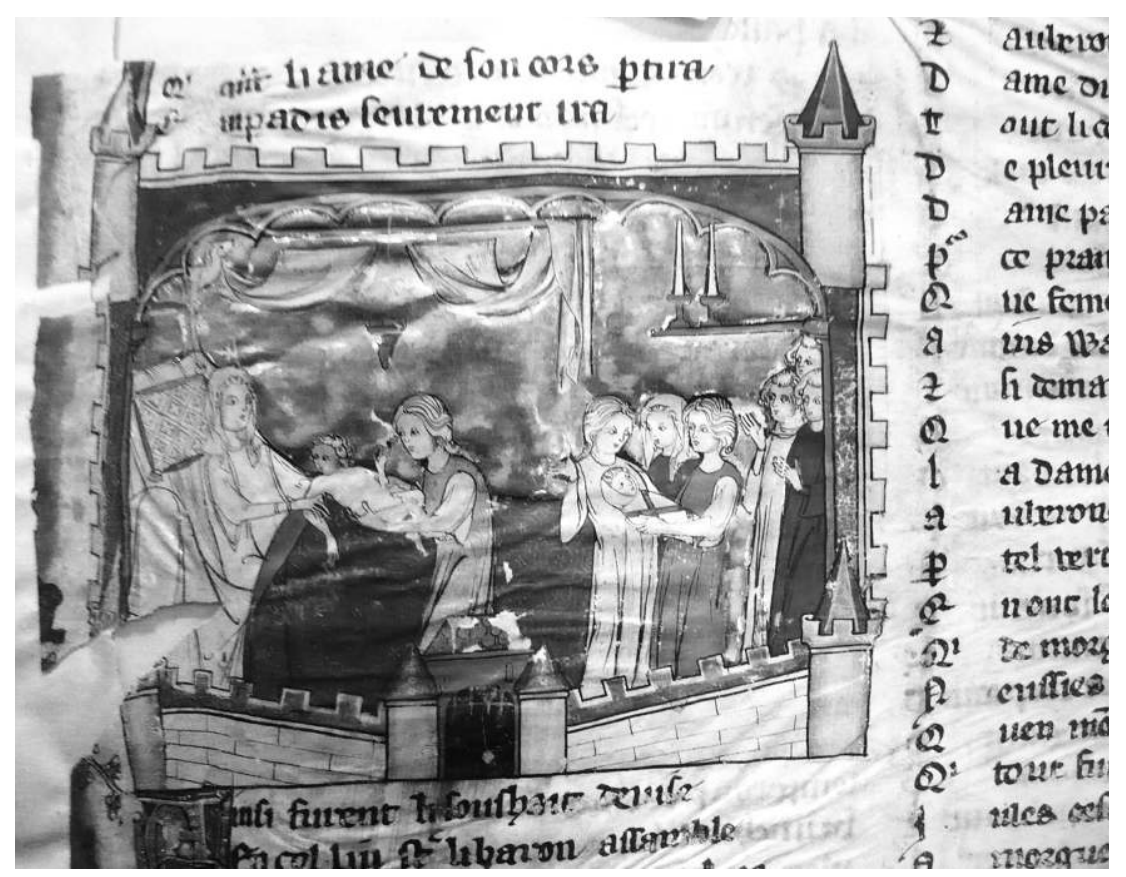

Fig. 4. Troisième miniature : naissance du roi Aubéron, les trois fées lui prodiguent leurs dons merveilleux (Torino, BNU, ms. L. II. 14, f. 291r).

Les fées sont respectivement vêtues de rose, de bleu et de rouge orangé afin de les différencier. Elles arborent des couleurs distinctes, attestant leur différence et des dons qu'elles s'apprêtent à offrir. Elles possèdent toutes des cheveux longs attachés, ce qui symbolise leur qualité ${ }^{51}$. L'uniformité de leur apparence contraste avec celle qu'on rencontre chez les hommes, plus diversifiée ${ }^{52}$. En effet, les personnages féminins de la miniature se ressemblent beaucoup, tant du point de vue physique que vestimentaire, et il serait aisé de les confondre.

Avec les nouveau-nés et leur mère, les fées constituent les personnages principaux de la miniature. Les quatre observateurs (la femme avec un voile sur la tête et trois hommes, possiblement des membres de la famille). Une des mains de deux de ces hommes semble signifier une expression de joie ou de surprise.

La scène représentée par le miniaturiste revêt une importance cruciale pour le récit : on comprend en particulier d'où viennent les attributs (féériques ou non) d'Aubéron, le principal héros du roman : «"Je li soushaide que puis ne

51. F. GARnier, Le Langage de l'image au Moyen âge..., p. 74.

52. J. Drobinsky, « La coiffure féminine entre moyen d'identification et principe axiologique dans l'iconographie de Guillaume de Machaut », dans C. Connochie-Bourgne éd., La Chevelure dans la littérature et l'art du Moyen Âge, Aix-en-Provence, 2004, p. 111. 
croistera / Que de hauteur. III. piés sans plus ara" » (v. 1398-1399). Autrement dit, les attributs des enfants peuvent témoigner du destin merveilleux des personnages. Par ailleurs, les deux enfants ont une très abondante chevelure bouclée. Cette inadéquation avec la réalité pourrait relever du caractère magique, probable lorsqu'il est question d'illustrations médiévales, des deux nouveau-nés. De plus, les deux chandeliers sur une tablette, en haut à droite de l'image, ont une fonction particulière, celle de symboliser la force du religieux et la lumière spirituelle, et cela pour chacun des jumeaux.

Les oiseaux sont de retour dans les marges du folio. Ils démontrent que :

Les motifs des marges ne fonctionnent pas seulement par référence au texte, mais aussi les uns par rapport aux autres, et ce renvoi des images entre elles opère à l'intérieur d'une même page comme aussi bien à travers une chaîne de motifs et de signes qui se répondent tout au long du livre ${ }^{53}$.

Ces éléments animaliers sont en accord avec l'événement heureux des naissances qui sont illustrées dans la miniature. Les deux scènes en bas de page, à la fin des colonnes de texte, sont probablement les marges les plus élaborées du roman ainsi que les plus porteuses de significations. Le personnage à droite est un homme presque nu, vêtu de lilas. Dans sa main droite, il tient un cor rouge qui traîne à terre ; dans sa main gauche, il a quelque chose ressemblant à un miroir ou à une boule de cristal (fig. 7). Ces deux objets pourraient concorder avec un personnage « faé ».

À droite, on aperçoit un personnage à cheval accompagné d'un chien et d'un homme âgé (fig. 5). Selon Jean Subrenat, il s'agit d'une scène de la Bible : «Le bas du folio 291 recto semble faire allusion à la "Fuite" de la Sainte Famille (un personnage féminin à cheval suivi d'un homme d'un certain âge $)^{54}$ ». Cependant, à la lumière d'une observation plus approfondie de ce bas de page, il est impossible de dire avec certitude qu'il s'agit bien de Jésus et de ses parents. En fait, on voit plutôt un personnage à cheval qui coupe sa cape à l'aide d'une épée pour en donner une partie à un pauvre. Il pourrait donc s'agir de saint Martin, dont il existe de multiples représentations dans lesquelles il accomplit ce geste, notamment dans le Livre d'heures d'Étienne Chevalier ou encore dans un vitrail de l'église Saint-Martin d'Étampes (Essonne).

Le mélange de religieux, de réel (historique) et de merveilleux dans les miniatures est à l'image du Roman d'Aubéron, qui met en place la généalogie de ce héros « si curieux et si attachant ${ }^{55}$ ». Si l'artiste a choisi ces trois scènes, c'est à la fois pour mettre l'emphase sur leur importance à

53. M. CAMILle, Images dans les marges..., p. 59.

54. J. SubrenAt, Le Roman d'Aubéron..., p. LXXV.

55. Ibid., p. XI. 


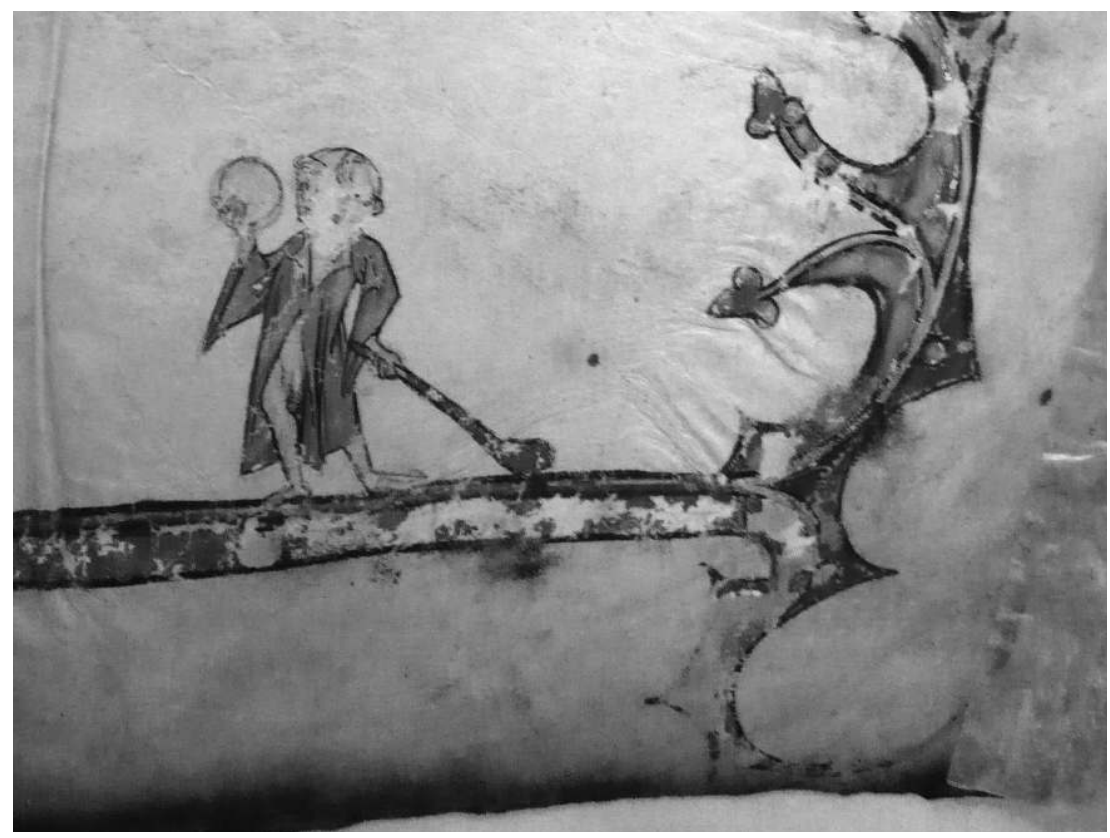

Fig. 7. Image marginale : personnage presque nu, vêtu de lilas, qui tient d'une main un cor rouge et de l'autre un miroir ou une boule de cristal (Torino, BNU, ms. L. II. 14, f. 291r).

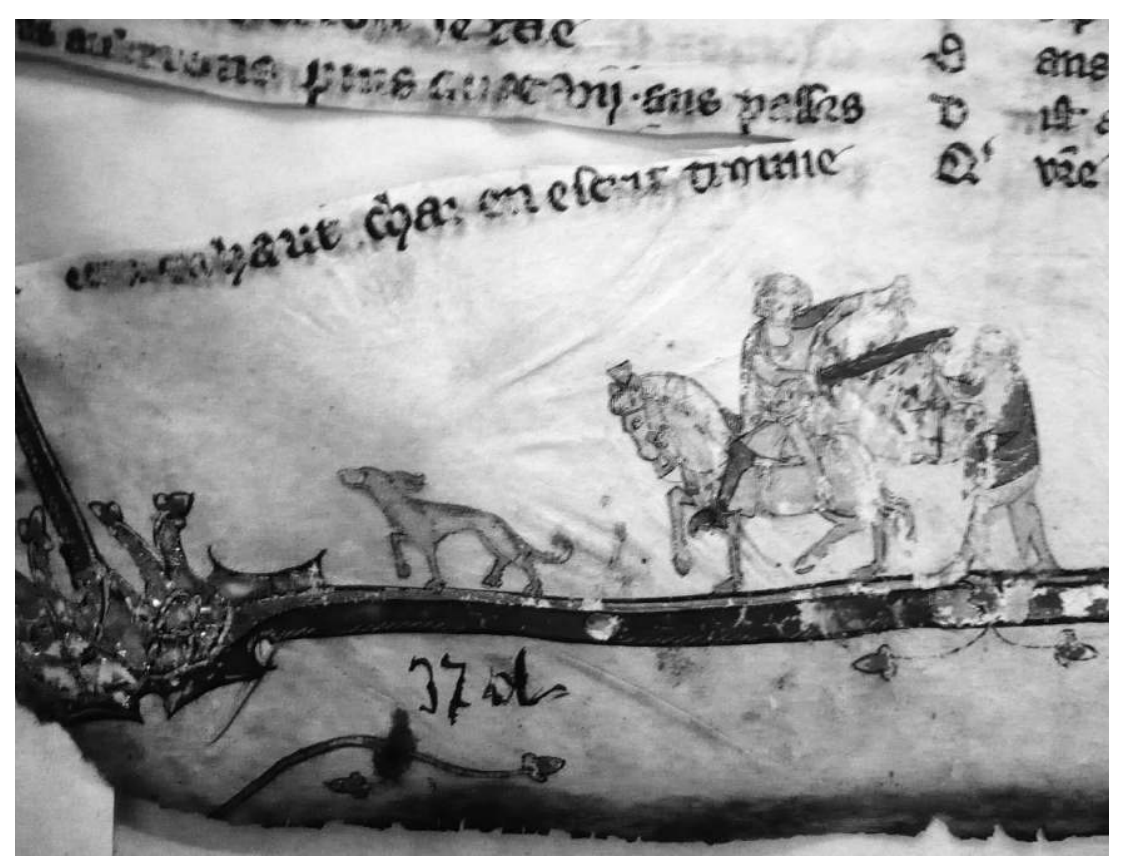

Fig. 5. Image marginale : saint Martin coupant sa cape (Torino, BNU, ms. L. II. 14, f. 291r). 
l'intérieur de l'histoire (ce qui vaut surtout pour la première et la troisième miniature) et pour faire valoir un fait d'armes impressionnant (deuxième miniature). Pour l'image du folio 283, l'artiste a choisi une division en triptyque de sorte que les éléments représentés constituent une mine d'informations précieuses. De plus, comme l'illustration, lisible de gauche à droite, représente d'abord la vision d'un songe, on comprend d'emblée l'importance à venir dans le récit d'éléments appartenant à un monde irréel. Dans le folio 289, un personnage « sacré » a le dessus sur un géant, c'està-dire un personnage merveilleux. Finalement, au folio 291, le personnage qui donne son nom au roman naît et se voit offrir des dons enchantés par un trio de fées. On peut donc dire que le réel et le surnaturel sont savamment entremêlés tout au long des miniatures, comme dans le texte.

Le manuscrit qui contient ce récit, le L. II. 14 de la Biblioteca nazionale universitaria de Turin, pourrait sembler moins intéressant vu son état de dégradation. Les miniatures sont cependant conservées dans un état plus qu'acceptable et se prêtent très bien à un exercice de lecture texte/ images. Il ne faut donc pas se laisser décourager par l'état du volume. Ces images nous permettent notamment de voir à quel point le miniaturiste est resté fidèle au roman et à ses enjeux.

Julie Grenon-Morin - Département des études littéraires - Université du Québec à Montréal

\section{Le Roman d'Aubéron. Une lecture texte/images}

Le manuscrit du Roman d'Aubéron, écrit entre 1260 et 1311 (et connu comme étant le prologue au cycle de Huon de Bordeaux), contient trois miniatures et des enluminures qui nourrissent son texte. II est conservé dans un imposant volume, à Turin, qui a partiellement brûlé en 1904. Néanmoins, les images demeurent visibles et signifiantes. La première est une composition en trois volets où se mêlent des faits militaires, la nature et un certain côté mystique. La deuxième est une scène de bataille esthétiquement très bien réussie et conservée où Jules César connaît le triomphe. La troisième représente la scène de la naissance des jumeaux Aubéron et Georges, alors que trois fées sont occupées à leur octroyer des vœux. Nous rendons ici compte de l'état des miniatures et des particularités des illustrations en lien avec le texte, afin de les décoder et de parfaire la compréhension de ce roman dont l'édition critique a été réalisée par Jean Subrenat en 1973.

Aubéron - féerie - manuscrit - marges - miniatures

\section{The Roman d'Aubéron : A Text/Image Reading}

The manuscript of the Roman d'Aubéron, written between 1260 and 1311 (and known as the prologue to the Huon de Bordeaux cycle), contains three miniatures and some illuminations that nourish the text. It is kept in imposing volume, in Torino, which partially burned in 1904. Nevertheless, the images stay visible and significant. The first one is a composition in three parts where are mixed military acts, nature and a certain mystical side. The second one is a battle scene aesthetically very well executed and preserved where Julius Caesar triumphs. The third one represents the birth scene of 
the twins Auberron and Georges, while the three fairies are busy to grant them with wishes. We give here an account of the miniature's condition and their particularities linked with the text, so we can decode them and perfect the understanding of this novel edited by Jean Subrenat in 1973.

Auberon - fairy-tale - manuscript - marginalia - miniatures

Crédit iconographique : (C) "Ministerio dei Beni e delle Attività Culturali e del Turismo, Biblioteca Nazionale Universitaria di Torino". Divieto di riproduzione. 